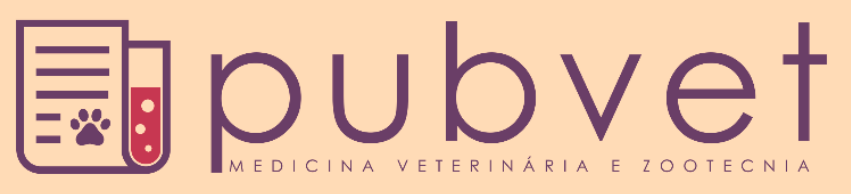

https://doi.org/10.31533/pubvet.v14n12a709.1-10

\title{
Zoneamento bioclimático para produção de bovinos de leite na região sudeste da Amazônia Legal
}

\author{
Kalebe Lima Guimarães ${ }^{10}$, Patricia Luyze da Costa Sarmento ${ }^{20}$, Maria Cristina Manno ${ }^{20}$, \\ Monique Valéria de Lima Carvalhal ${ }^{*}$ (9)
}

${ }^{I}$ Faculdade de Ensino Superior da Amazônia Reunida (FESAR), Redenção-Pará, Brasil.

${ }^{2}$ Instituto da Saúde e Produção Animal - Universidade Federal Rural da Amazônia (UFRA), Belém-Pará, Brasil.

*Autor para correspondência, e-mail: monique_carvalhal@hotmail.com

\begin{abstract}
Resumo. O objetivo desse trabalho foi realizar o zoneamento bioclimático da região do Sudeste do Pará visando à produção de vacas leiteiras. Para a realização do zoneamento bioclimático foram coletados dados dos anos de 1992 a 2017, das estações meteorológicas convencionais de responsabilidade do Instituto Nacional de Meteorologia (INMET) que compreendem a região Sudeste do Pará. Com os dados observados neste estudo é possível afirmar que as condições climáticas da região estudada não são ideais para a produção de animais das raças Jersey, Holandês e Girolando, apresentando temperatura e umidade relativa do ar constantemente elevadas e com o índice de temperatura e umidade (ITU) acima de 78. Dessa forma, reforçamos a necessidade da utilização de modificações ambientais nas propriedades produtivas da região. Incentivamos ainda iniciativas que visem conhecer as estratégias adaptativas dos bovinos leiteiros em condição de estresse térmico.
\end{abstract}

Palavras-chave: Bem-estar animal, conforto térmico, índice de temperatura e umidade

\section{Bioclimatic zoning for dairy cattle production in the southeast region of the Legal Amazon}

\begin{abstract}
This study intended to establish the bioclimatic zoning of the southeast state of Pará aiming at the production of dairy cows. For the realization of bioclimatic zoning, data from the years 1992 to 2017 were collected from conventional meteorological stations under the responsibility of the National Meteorological Institute (INMET) that comprise the Southeast region of Pará. With the data observed in this study, it is possible to affirm that the climatic conditions of the studied region are not ideal for the production of animals of the Jersey, Holstein and Girolando breeds, presenting constant high temperature and relative humidity and with the temperature and humidity index (THI) above 78. Thus, we reinforce the need to use environmental changes in the productive farms of the region. We also encourage studies that aim to understand the adaptive strategies of dairy cattle under thermal stress conditions.
\end{abstract}

Keywords: Eelfare, thermal comfort, temperature and humidity index

\section{Zonificación bioclimática para la producción de ganado lechero en la región sureste de la Amazonía Legal}

Resumen. El objetivo de este trabajo fue llevar a cabo la zonificación bioclimática de la región sudeste de Pará con el objetivo de producir vacas lecheras. Para la realización de la zonificación bioclimática, se recopilaron datos de los años 1992 a 2017 de estaciones meteorológicas convencionales bajo la responsabilidad del Instituto Nacional de Meteorología (INMET) que comprende la región sudeste de Pará. Con los datos observados 
en este estudio, es posible establecer que las condiciones climáticas de la región estudiada no son ideales para la producción de animales de las razas Jersey, Holstein y Girolando, presentando temperaturas y humedad relativa constantemente elevadas y con un índice de temperatura y humedad (ITU) superior a 78. De esta forma, reforzamos la necesidad de aprovechar los cambios ambientales en las propiedades productivas de la región. También fomentamos iniciativas que tengan como objetivo comprender las estrategias de adaptación del ganado lechero bajo estrés térmico.

Palabras clave: Bienestar animal, confort térmico, índice de temperatura y humedad

\section{Introdução}

A pecuária de leite tem apresentado notável crescimento na Amazônia nas últimas décadas, impulsionada pelos subsídios do governo federal em meados da década de oitenta e pelo processo de expansão da fronteira amazônica e sua inclusão em um mercado globalizado devido à demanda por produtos agropecuários. Políticas de ocupação territorial como a construção de rodovias e planos de colonização impulsionaram o surgimento das bacias leiteiras formadas sobretudo por propriedades de agricultura familiar. O Pará, segundo maior estado brasileiro em extensão, ocupa a décima colocação em produção de leite no país e a segunda maior produção da região Norte, com 33,9\% do total produzido na região (Soares et al., 2019; Sena, 2010). Embora praticada em todo o estado, a bovinocultura de leite se mostra mais expressiva na região do Sudeste paraense. O estado possui seis mesorregiões, sendo a sudeste composta por 39 municípios dentre os quais estão os dez com maior produção de leite do estado (IBGE, 2017; Santos, 2011). Apesar dos índices numéricos indicarem elevada produção de leite, a produtividade do estado (produção de litros/vaca/ano) é baixa em relação a outros estados brasileiros. Sendo assim, adequação tecnológica da cadeia produtiva do leite, assume um papel importante para desenvolvimento do setor nessa região. A busca por sistemas de criação mais produtivos e compatíveis com as condições ambientais predominantes no estado é uma preocupação constante de pesquisadores, produtores e técnicos (Ferreira et al., 2001).

As características climáticas representam um dos fatores que podem ser considerados limitantes para produtividade leiteira. A região amazônica apresenta elevadas temperaturas e pluviosidade, ausência de estação fria e níveis de insolação considerados altos (Nogueira, 2010). A associação entre elevadas temperaturas, alta umidade do ar e radiação solar pode acarretar alterações comportamentais e fisiológicas em diferentes espécies leiteiras (Alam et al., 2013; Dash et al., 2016; West et al., 2003). Borburema et al. (2013), aponta efeitos do ambiente como modificadores da condição fisiológica das vacas leiteiras, que resultarão em baixos índices produtivos, pois com o aumento dos parâmetros fisiológicos há diminuição da ingestão de alimentos e consequentemente, redução na produção de leite, baixas taxas de concepção e atraso no crescimento de animais de reposição, ocasionando perdas econômicas significativas para o produtor.

A escolha da raça é outro fator determinante para produtividade dos sistemas leiteiros, pois está diretamente relacionada com a adaptação do animal ao clima da região. As vacas de raças especializadas para produção leiteira, em condição de estresse térmico, geralmente apresentam produtividade reduzida em razão do consumo de energia para manutenção da temperatura corporal e menor consumo de alimento (Rodrigues et al., 2017). Neste contexto, há uma tendência crescente do uso de cruzamentos entre Bos taurus e Bos indicus para exploração leiteira em regiões tropicais, de forma a combinar a capacidade produtiva do primeiro com a rusticidade do segundo (Costa et al., 2015). Assim, para que os animais possam manifestar todo o seu potencial produtivo, torna-se necessário considerar a interação entre a genética e o ambiente térmico.

Devido ao potencial produtivo da bovinocultura leiteira na região Amazônica e a possibilidade de crescimento, torna-se necessário o desenvolvimento de pesquisas na área do bem-estar animal, principalmente na realização de um zoneamento bioclimático para esses animais. $\mathrm{O}$ zoneamento bioclimático, é uma ferramenta que através do monitoramento das condições climáticas, permite a previsão de regiões e/ou épocas com maior possibilidade de ocorrência do estresse térmico. Essa ferramenta facilita no momento de decisão referente ao manejo ambiental que deve ser adotado para minimizar o estresse ocasionado pelo clima. 
Dessa forma, considerando a influência dos fatores climáticos na produção de leite das principais raças criadas na região sudeste paraense, o objetivo desse trabalho foi realizar o zoneamento bioclimático para vacas leiteiras da região do Sudeste do Pará a partir dos valores do Índice de Temperatura e Umidade (ITU).

\section{Material e métodos}

Para gerar o zoneamento bioclimático foram coletados dados dos anos de 1992 a 2017, das estações meteorológicas convencionais de responsabilidade do INMET que compreendem a região sudeste paraense, distribuídas nos municípios de Marabá, São Felix do Xingu, Tucuruí e Conceição do Araguaia, fornecidos pelo Instituto Nacional de Meteorologia (INMET). As estações são compostas por vários sensores isolados responsáveis por registar continuamente os parâmetros meteorológicos como temperatura, umidade relativa do ar, precipitação, radiação solar, direção e velocidade do vento. Esses dados são aferidos por um observador a cada intervalo de tempo (As 00:00h, 06:00h, 12:00h e 18:00h), que os envia a um centro coletor de dados do INMET.

Este trabalho foi desenvolvido a partir de dados climáticos obtidos gerados pelo registro contínuo dos parâmetros meteorológicos como temperatura, umidade relativa do ar, precipitação, radiação solar, direção e velocidade do vento por estações meteorológicas convencionais do INMET. Para a composição do zoneamento foram utilizadas as seguintes variáveis climáticas: Média da temperatura do ar (Tméd); Média das máximas da temperatura do ar (Tmáx); Média dos mínimos da temperatura do ar (Tmín); Média da umidade relativa (URméd); Média das máximas da umidade relativa (URmáx); Média das mínimas da umidade relativa (URmín); Média do Índice de Temperatura e Umidade (ITU).

Para a realização do cálculo, foram utilizadas as médias das temperaturas e da umidade relativa (mensal) de todos os anos. Existem vários índices que podem medir de forma ampla o efeito do estresse sobre os animais. Entretanto, para este trabalho optou-se por utilizar o ITU por conta da disponibilidade de dados por parte do INMET.

O Índice de Temperatura e Umidade (ITU) foi determinado pela seguinte equação:

$\mathrm{ITU}=0,8 \mathrm{Ta}+\mathrm{UR}(\mathrm{Ta}-14,3) / 100+46,3$, onde: Ta - temperatura de bulbo seco $\left({ }^{\circ} \mathrm{C}\right) ; \mathrm{UR} \%-$ umidade relativa do ar $(\%)$.

A análise dos dados para obtenção das medias que compõem o zoneamento bioclimático foi realizada no programa Microsoft office Windows Excel $^{\circledR}$ versão 2016. Estes valores foram utilizados para comparar com as condições de conforto térmico ideais para vacas em lactação das diferentes raças de acordo com dados de literatura. Para comparar as exigências dos animais com os valores climáticos da microrregião, foi adotada a seguinte simbologia: I - Inferiores aos exigidos para vacas em lactação; C confortáveis aos exigidos para vacas e; $\mathrm{S}$ - superiores aos exigido de acordo com a literatura. Para o ITU foi adotado a seguinte legenda: 1 (Condição ambientais normais), 2 (Condições de estresse críticas), 3 (Condição de perigo). As variações mensais dos fatores climáticos, conforme recomendadas na literatura, estão descritas na Tabela 1.

Tabela 1. Valores de referências para os critérios zona de conforto térmico (ZCT), Temperatura critica inferior (TCI), Temperatura critica superior (TCS), Umidade relativa do ar (UR), Índice de conforto térmico (ITU) em condições normais, de estresse e em perigo para vacas em lactação das raças Holandês, Jersey e Girolando.

\begin{tabular}{|c|c|c|c|c|}
\hline \multirow{2}{*}{$\begin{array}{l}\text { Variáveis } \\
\text { climáticas }\end{array}$} & \multicolumn{3}{|c|}{ Raças } & \multirow{2}{*}{ Autores } \\
\hline & Holandês & Jersey & Girolando & \\
\hline$\overline{\mathrm{ZCT}}$ & 5 a 21 & 5 a 24 & 5 a 26 & Hafez et al. (1973); Curtis (1983); Perissinotto et al. (2009) \\
\hline TCI & -10 & -10 & $\mathrm{NE}^{*}$ & Hafez et al. (1973); Curtis (1983) \\
\hline TCS & 27 & 27 & 31 & Johnson (1965); Azevedo et al. (2005); Perissinotto et al. (2009) \\
\hline UR $(\%)$ & $\begin{array}{c}<70 \% \\
50 \%-70 \% \\
40 \text { a } 70 \%\end{array}$ & $\begin{array}{c}<70 \% \\
50 \%-70 \% \\
40 \text { a } 70 \%\end{array}$ & $\begin{array}{c}<70 \% \\
50 \%-70 \% \\
40 \text { a } 70 \%\end{array}$ & $\begin{array}{l}\text { Nääs \& Arcaro Júnior (2001); Matarazzo et al. (2007); Ferreira } \\
\text { (2011); Mollo Neto et al. (2014) }\end{array}$ \\
\hline ITU normal & $<72$ & $<70$ & $<72$ & Perissinotto \& Moura (2007) \\
\hline ITU estresse & $72-77$ & $72-74$ & $>72$ & $\begin{array}{l}\text { Damasceno et al. (1998); Silva et al. (2002); Azevedo et al. } \\
\text { (2005); Perissinotto \& Moura (2007); Smith et al. (2013) }\end{array}$ \\
\hline ITU perigo & $>77$ & $>77$ & $>79$ & $\begin{array}{l}\text { Davis et al. (2003); Fuquay (1981); Azevedo et al. (2005); } \\
\text { Perissinotto \& Moura (2007) }\end{array}$ \\
\hline
\end{tabular}

*NE: Informação não encontrada na literatura cientifica. 


\section{Resultados e discussão}

Conhecer os parâmetros climáticos e a oscilação desses no decorrer do ano pode auxiliar as tomadas de decisão e minimizar os prejuízos produtivos ocasionados pelo clima. Nesse estudo foi realizada a caracterização do ambiente térmico na região sudeste paraense visando à produção leiteira. A Tabela 2 mostra os valores médios das variáveis climáticas coletados pelas estações meteorológicas localizadas na região sudeste paraense durante os anos de 1992 a 2017.

O estado do Pará caracteriza-se pela ocorrência do Clima Equatorial (Luz et al., 2013), sendo caracterizado por elevadas temperaturas médias do ar e pluviosidade e as estações do ano não são bem definidas.

Tabela 2. Valores médios mensais para temperatura média (Tméd), mínima (Tmín), e máxima (Tmáx) em graus Celsius $\left({ }^{\circ} \mathrm{C}\right)$, umidade relativa do ar média (URmed), mínima (URmín) e máxima (URmáx), dada em porcentagem, e Índice de Temperatura e Umidade (ITU) na Região Sudeste do estado do Pará, Brasil.

\begin{tabular}{|c|c|c|c|c|c|c|c|c|c|c|c|c|}
\hline \multirow{2}{*}{ Variáveis } & \multicolumn{12}{|c|}{ Meses do ano } \\
\hline & Jan & $\mathrm{Fev}$ & Mar & Abr & Mai & Jun & Jul & Ago & Set & Out & Nov & Dez \\
\hline Tmed & 26 & 26 & 26 & 27 & 28 & 28 & 28 & 28 & 28 & 28 & 27 & 27 \\
\hline Tmax & 32 & 32 & 32 & 32 & 33 & 34 & 34 & 35 & 35 & 34 & 33 & 32 \\
\hline Tmin & 21 & 21 & 24 & 23 & 23 & 25 & 21 & 22 & 23 & 24 & 23 & 23 \\
\hline URMed & 85 & 86 & 86 & 85 & 82 & 76 & 71 & 70 & 73 & 77 & 81 & 83 \\
\hline URMax & 92 & 93 & 92 & 92 & 90 & 88 & 85 & 86 & 87 & 88 & 90 & 92 \\
\hline URMin & 79 & 78 & 81 & 80 & 76 & 68 & 60 & 59 & 61 & 68 & 74 & 78 \\
\hline ITU & 78 & 77 & 78 & 78 & 80 & 79 & 78 & 78 & 79 & 79 & 79 & 78 \\
\hline
\end{tabular}

Fonte: tabulação de dados do INMET - $2^{\circ}$. DISME, dos anos de 1992 a 2017.

Nesse estudo, o Zoneamento Bioclimático foi definido com base no modelo de Sarmento (2019) em que Temperatura do Ar e Umidade Relativa foram classificadas pelas letras I (Inferior), C (Conforto) e $\mathrm{S}$ (Superior) quando estiverem respectivamente inferiores, dentro ou superiores à Zona de conforto térmico. Para classificar o ITU foram utilizados números em que 1 caracteriza condição normal, 2 condições de alerta de estresse, 3 condições de perigo.

Tabela 3. Zoneamento Bioclimático da região sudeste paraense com base nas Médias de Temperaturas, Umidade Relativa do Ar indicadas pelas letras I (Inferior), C (Conforto) e S (Superior) quando estiverem respectivamente inferiores, dentro ou superiores à Zona de conforto térmico para bovinos da raça holandesa. Para a classificação do ITU foram utilizados números em que 1 caracteriza condição normal, 2 condições de alerta de estresse, 3 condições de perigo.

\begin{tabular}{|c|c|c|c|c|c|c|c|c|c|c|c|c|}
\hline \multicolumn{13}{|c|}{ Raça Holandesa } \\
\hline \multirow{2}{*}{ Variáveis } & \multicolumn{12}{|c|}{ Meses do ano } \\
\hline & Jan & $\mathrm{Fev}$ & Mar & Abr & Mai & Jun & Jul & Ago & Set & Out & Nov & Dez \\
\hline Tmed & $\mathrm{S}$ & $\mathrm{S}$ & $\mathrm{S}$ & $\mathrm{S}$ & $\mathrm{S}$ & $\mathrm{S}$ & $\mathrm{S}$ & $\mathrm{S}$ & $\mathrm{S}$ & $\mathrm{S}$ & $\mathrm{S}$ & $\mathrm{S}$ \\
\hline Tmax & S & $S$ & $\mathrm{~S}$ & $S$ & $\mathrm{~S}$ & $S$ & $S$ & $S$ & $S$ & $S$ & $S$ & $\mathrm{~S}$ \\
\hline Tmin & $\mathrm{C}$ & $\mathrm{C}$ & $S$ & $S$ & $S$ & $S$ & $\mathrm{C}$ & $S$ & $S$ & $S$ & S & $\mathrm{S}$ \\
\hline URMed & S & S & $\mathrm{S}$ & $S$ & $S$ & S & S & $\mathrm{C}$ & $S$ & $S$ & S & $S$ \\
\hline URMax & $S$ & $S$ & $S$ & $S$ & $S$ & $S$ & $S$ & $S$ & $S$ & $S$ & $S$ & $S$ \\
\hline URMin & $S$ & $S$ & $S$ & $S$ & $S$ & $\mathrm{C}$ & $\mathrm{C}$ & $\mathrm{C}$ & $\mathrm{C}$ & $\mathrm{C}$ & $S$ & $S$ \\
\hline ITU & 3 & 2 & 3 & 3 & 3 & 3 & 3 & 3 & 3 & 3 & 3 & 3 \\
\hline
\end{tabular}

A raça holandês é considerada especializada para produção leiteira, no entanto, são dependentes de temperatura amena (Passetti et al., 2016). As temperaturas do ar observadas ao longo do ano na região sudeste paraense são superiores as temperaturas máximas suportadas por essa raça. Apesar das temperaturas críticas do ar não serem características fixas para nenhuma espécie ou tipo de animal, ou seja, podem mudar com a idade e condições fisiológicas, Nascimento et al. (2017), descreveram que para raça holandesa a temperatura do ar é uma variável que afeta diretamente a produção de leite qualquer estágio da fase de lactação.

A raça Jersey também é considerada uma raça especializada na produção leiteira, sendo comumente utilizada na região sul do Brasil (Soares et al., 2011). O clima observado na região sul é classificado 
como temperado (grupo C da classificação Köppen), o que pode ser considerado um fator determinante para adaptação dessa raça. Nossos resultados mostram que na região sudeste do Pará esses animais são expostos, ao longo do ano, a temperaturas superiores a máxima suportada pela raça, como mostra a Tabela 4.

A qualidade do leite de animais da raça Jersey é considerada superior quando comparada com outras raças, esses animais apresentam teores de sólidos totais elevados no leite. No entanto, vacas da raça Jersey apresentam queda na produção de gordura, proteína e ácidos graxos de cadeia curta sob condições de estresse térmico, além de queda no desempenho reprodutivo, ocasionando perdas de pelo menos \$ 123-164 dólares por animal em razão do maior tempo para idade ao primeiro parto nos Estados Unidos (Bandaranayaka \& Holmes, 1976; Teke \& Akdag, 2012). A temperatura média e máxima do ar observadas na região se manteve por todo ano superior ao limite máximo da zona de conforto térmico para as raças Holandesas e Jersey. Quando a temperatura do ar permanece acima da máxima suportada pela espécie, os mecanismos de termorregulação são iniciados visando à dissipação do calor. Se não houver diminuição da temperatura do ambiente os mecanismos de termorregulação são intensificados ocasionando maior gasto energético, resultando em prejuízos produtivos (Silva, 2000).

Tabela 4. Zoneamento Bioclimático da região sudeste paraense com base nas Médias de Temperaturas, Umidade Relativa do Ar indicadas pelas letras I (Inferior), C (Conforto) e S (Superior) quando estiverem respectivamente inferiores, dentro ou superiores à Zona de conforto térmico para bovinos da raça Jersey. Para a classificação do ITU foram utilizados números em que 1 caracteriza condição normal, 2 condições de alerta de estresse, 3 condições de perigo.

\begin{tabular}{|c|c|c|c|c|c|c|c|c|c|c|c|c|}
\hline \multicolumn{13}{|c|}{ Raça Jersey } \\
\hline \multirow{2}{*}{ Variáveis } & \multicolumn{12}{|c|}{ Meses do ano } \\
\hline & Jan & Fev & Mar & Abr & Mai & Jun & Jul & Ago & Set & Out & Nov & Dez \\
\hline Tmed & $\mathrm{S}$ & $\mathrm{S}$ & $\mathrm{S}$ & $\mathrm{S}$ & $\mathrm{S}$ & $\mathrm{S}$ & $\mathrm{S}$ & $\mathrm{S}$ & $\mathrm{S}$ & $\mathrm{S}$ & $\mathrm{S}$ & $\mathrm{S}$ \\
\hline $\operatorname{Tmax}$ & $S$ & $S$ & S & $S$ & $S$ & S & S & S & $S$ & $S$ & $S$ & $S$ \\
\hline Tmin & $\mathrm{C}$ & $\mathrm{C}$ & $\mathrm{C}$ & $\mathrm{C}$ & $\mathrm{C}$ & S & $\mathrm{C}$ & $\mathrm{C}$ & $\mathrm{C}$ & $\mathrm{C}$ & $\mathrm{C}$ & $\mathrm{C}$ \\
\hline URMed & S & S & S & S & S & S & $S$ & S & $S$ & S & S & S \\
\hline URMax & S & S & S & $S$ & $S$ & S & $S$ & S & $S$ & S & S & $S$ \\
\hline URMin & $S$ & $S$ & $S$ & $S$ & $S$ & $\mathrm{C}$ & $\mathrm{C}$ & $\mathrm{C}$ & $\mathrm{C}$ & $\mathrm{C}$ & $S$ & $S$ \\
\hline ITU & 3 & 3 & 3 & 3 & 3 & 3 & 3 & 3 & 3 & 3 & 3 & 3 \\
\hline
\end{tabular}

A umidade relativa do ar também é uma variável climática determinante na adaptação dos animais em clima tropical. Nossos resultados mostram que na maioria dos meses do ano a umidade do ar também é considerada superior ao máximo permitido para as vacas em lactação das raças Jersey e Holandesa. Esta variável climática está diretamente relacionada com a capacidade do animal trocar calor com o ambiente, sendo assim, a alta umidade reduz o potencial de evaporação respiratória e da pele do animal (sudação), piorando os efeitos da temperatura elevada (Silva et al., 2007). Sota (1996) descreve que em condições de umidade elevada, o ar saturado irá inibir a evaporação da água pela pele e sistema respiratório, proporcionando para os animais maior dificuldade de dissipar calor.

O ITU tem sido amplamente utilizado para avaliar ambientes de produção de animais, mesmo em regiões tropicais, apesar de suas limitações, pois conseguem quantificar, em uma única variável, os efeitos do estresse térmico sofrido pelos animais. De maneira geral, considera-se que as vacas leiteiras começam a sofrer os efeitos do estresse térmico sempre que o ITU ultrapassar 72 (Baêta \& Souza, 2010). Nossos resultados mostram que as vacas em lactação das raças Jersey e Holandesa criadas na região sudeste paraense permanecem, ao longo do ano, em condição de perigo em relação ao ITU. Dessa forma, temperatura elevada, variações climáticas acentuadas, como oscilações na umidade relativa do ar não podem ser consideradas condições adequadas para a produção de vacas leiteiras.

Vale destacar que os índices climáticos são mecanismos uteis para avaliar o clima geral de uma área, mas as variáveis e seus coeficientes em um determinado índice devem ser consistentes com os mecanismos fisiológicos de troca de calor dos animais, nesse estudo não avaliamos as respostas fisiológicas das vacas. Além disso, consideramos que a radiação solar é uma variável em criações tropicais que não pode ser ignorada, por isso, reforçamos a necessidade de disponibilização desses dados pelo Instituto Nacional de Meteorologia. 
De modo geral, bovinos do tronco genético Bos taurus apresentam redução na ingestão de alimentos sob condições de estresse térmico e têm sua produtividade prejudicada, pois produzem maior calor metabólico para produção de leite em relação a vacas de média produtividade. Sendo indicada a estratégia de cruzamento direcionado entre o gado de origem europeu e zebu, já que estas últimas apresentam maior zona de conforto térmico e limites de ITU mais altos (Breihnholt et al., 1981). Dentre esses cruzamentos destacam-se aqueles produzidos com a raça Gir, que são predominantes nos rebanhos leiteiros brasileiros, evidenciando a raça Girolando, que contribuiu por $80 \%$ do leite produzido no Brasil (Madalena et al., 2012). Na Tabela 5 é apresentando o zoneamento bioclimático da região sudeste paraense para os animais da raça Girolando.

A raça Girolando quando comparada com as raças puras é considerada mais adaptada às condições climáticas do ambiente tropical. Das três raças avaliadas nesse estudo, a Girolando pode ser considerada a raça mais adaptada, considerando as médias de temperatura mínima observadas. No entanto, as temperaturas mínimas não representam a totalidade da condição ambiental observada na região. Sendo assim, esses animais também sofrem por estresse térmico na região sudeste paraense ao longo do ano, tendo em vista as temperaturas médias, máximas, umidade relativa do ar e o valor de ITU que indica alerta de estresse. Borges et al. (2012) descreveram que animais frutos do cruzamento (Holandês x Gir) também apresentam estresse por incremento calórico, sobretudo nos cruzamentos em que a composição genética Holandês estiver em maior proporção. Dessa forma, também é importante avaliar a composição racial desses animais.

Tabela 5. Zoneamento Bioclimático da região sudeste paraense com base nas Médias de Temperaturas e Umidade Relativa do Ar indicadas pelas letras I (Inferior), C (Conforto) e S (Superior) quando estiverem respectivamente inferiores, dentro ou superiores à Zona de conforto térmico para bovinos da raça Girolando. Para a classificação do ITU foram utilizados números em que 1 caracteriza condição normal, 2 condições de alerta de estresse, 3 condições de perigo.

\begin{tabular}{|c|c|c|c|c|c|c|c|c|c|c|c|c|}
\hline \multicolumn{13}{|c|}{ Raça Girolando } \\
\hline \multirow{2}{*}{ Variáveis } & \multicolumn{12}{|c|}{ Meses do ano } \\
\hline & Jan & Fev & Mar & Abr & Mai & Jun & Jul & Ago & Set & Out & Nov & Dez \\
\hline Tmed & $\mathrm{C}$ & $\mathrm{C}$ & $\mathrm{C}$ & $\mathrm{S}$ & $\mathrm{S}$ & $\mathrm{S}$ & $\mathrm{S}$ & $\mathrm{S}$ & $\mathrm{S}$ & $\mathrm{S}$ & $\mathrm{S}$ & $\mathrm{S}$ \\
\hline Tmax & $S$ & $\mathrm{~S}$ & $S$ & $\mathrm{~S}$ & $S$ & $\mathrm{~S}$ & $S$ & $\mathrm{~S}$ & $S$ & $\mathrm{~S}$ & $S$ & $\mathrm{~S}$ \\
\hline Tmin & $\mathrm{C}$ & $\mathrm{C}$ & $\mathrm{C}$ & $\mathrm{C}$ & $\mathrm{C}$ & $\mathrm{C}$ & $\mathrm{C}$ & $\mathrm{C}$ & $\mathrm{C}$ & $\mathrm{C}$ & $\mathrm{C}$ & $\mathrm{C}$ \\
\hline URMed & S & $\mathrm{S}$ & $\mathrm{S}$ & $S$ & S & $S$ & $S$ & $S$ & $\mathrm{~S}$ & $S$ & $S$ & S \\
\hline URMax & $\mathrm{S}$ & $S$ & $\mathrm{~S}$ & $S$ & S & $\mathrm{S}$ & $S$ & $S$ & S & $S$ & $S$ & $S$ \\
\hline URMin & $S$ & $\mathrm{~S}$ & $\mathrm{~S}$ & $S$ & $\mathrm{~S}$ & $\mathrm{C}$ & $\mathrm{C}$ & $\mathrm{C}$ & $\mathrm{C}$ & $\mathrm{C}$ & $S$ & $\mathrm{~S}$ \\
\hline ITU & 2 & 2 & 2 & 2 & 3 & 2 & 2 & 2 & 2 & 2 & 2 & 2 \\
\hline
\end{tabular}

O aprimoramento das técnicas de melhoramento genético no Brasil proporciona o aumento da produção de leite anual na raça Girolando, no entanto, a correlação entre o efeito genético aditivo para produção de leite e a tolerância ao calor é negativa (Finocchiaro et al., 2005). Além disso, animais mais produtivos aumentam a ingestão alimentar, o que resulta em maior produção de calor metabólico. Portanto, a tolerância ao calor desses animais pode ser reduzida com o aumento da produtividade ao longo dos anos. Dessa forma, destacamos a importância de estudos que visem avaliar a adaptabilidade e a tolerância ao calor das vacas leiteiras no Brasil e principalmente na região sudeste paraense.

Os mecanismos pelo qual o estresse afeta a produção dos animais é bastante detalhado na literatura científica. Para os animais leiteiros são relatados problemas que incluem alterações na quantidade e qualidade do leite (Bertocchi et al., 2014; Smith et al., 2013). Além disso, sob condições estressantes prolongadas ou extremas, o estresse pode resultar em problemas de saúde, perdas irreversíveis de produtividade ou até mesmo na morte do animal (Dobson \& Smith, 2000). Dessa forma, conhecer o ambiente térmico e entender a capacidade de adaptação das raças leiteiras exploradas no Brasil pode tornar a pecuária leiteira mais produtiva.

Diversas medidas podem ser adotadas para adequar às condições do ambiente de criação para gado leiteiro seja em sistemas de pastejo, galpões ou nas estruturas de ordenha. As modificações ambientais constituem o manejo do ambiente para esses animais. A utilização de sombreamento é modificação ambiental primária mais recomendada, com arborização em piquetes e no entorno da ordenha. Podendo ser adotado também o sistema silvipastoril onde há disposição racional de árvores nos piquetes com a 
finalidade adicional de exploração madeireira. Além do custo/benefício, o fornecimento de sombra pode reduzir o estresse térmico dos animais de 30\% a 50\% (Collier, 2006). Com as condições ambientais apresentadas nesse estudo, as árvores devem ser consideradas obrigatórias em piquetes para as vacas em lactação das raças Holandesa, Jersey e Girolando. O sombreamento artificial também é indicado para diminuir a insolação direta. Porém, deve sempre ser analisada sua eficiência em função do projeto, pois geralmente estes não apresentam melhoria na umidade do ar, tendo menor benefício para o microclima (Dhiman \& Zaman, 2001; Pires \& Campos, 2004).

Há também diversos sistemas de resfriamento que podem ser utilizados, como o resfriamento do próprio ar ambiental e diretamente do animal, por meio de ventiladores, e indiretamente pelo resfriamento dos elementos construtivos, como as coberturas (Baêta \& Souza, 2010), sobretudo nas salas de espera e saída da ordenha. Além dessas, outras intervenções apresentam efeitos positivos no microclima quando feitas em conjunto como a pintura de branco das superfícies externas, aspersão na cobertura de estruturas e uso de materiais com isolamento térmico. Orientações devem ser tomadas com base nas características locais de cada propriedade para melhor utilização das condições naturais e redução de custos (Pires \& Campos, 2004).

Com a expansão da pecuária leiteira no sudeste paraense, as mudanças relacionadas ao mercado consumidor mais competitivos e exigentes e a busca por sistemas produtivos mais sustentáveis na região Amazônica é necessário compreender as especificidades da atividade, visando mudanças no processo produtivo que representem ganhos de produtividade e sejam sustentáveis do ponto de vista social, ambiental e econômico.

\section{Conclusões}

O zoneamento bioclimático é importante para a estruturação de projetos na pecuária leiteira. Com os dados observados neste estudo é possível afirmar que as condições climáticas da região estudada não são ideais para a produção de vacas leiteiras das raças Jersey, Holandês e Girolando, apresentando temperatura e umidade relativa do ar constantemente elevadas e com uma média anual do valor de Índice de Temperatura e Umidade igual a 78, acima do ideal para as raças.

O estresse térmico em bovinos de leite influencia diretamente na produção, reprodução e pode acarretar prejuízos econômicos ao produtor. Sendo esta uma atividade de grande importância socioeconômica, e com base nos resultados encontrados, reforçamos a necessidade da utilização de modificações ambientais nas propriedades produtivas da região. Incentivamos ainda iniciativas que visem conhecer as estratégias adaptativas dos bovinos leiteiros em condição de estresse térmico.

\section{Referências bibliográficas}

Alam, M., Hashem, M., Rahman, M., Hossain, M., Haque, M., Sobhan, Z., \& Islam, M. (2013). Effect of Heat Stress on Behavior, Physiological and Blood Parameters of Goat. Progressive Agriculture, 22(1-2), 37-45. https://doi.org/10.3329/pa.v22i1-2.16465

Azevedo, M., Pires, M. F. Á., Saturnino, H. M., Lana, Â. M. Q., Sampaio, I. B. M., Monteiro, J. B. N., \& Morato, L. E. (2005). Estimativa de níveis críticos superiores do índice de temperatura e umidade para vacas leiteiras ${ }^{1 / 2}, 3 / 4$ e 7/8 Holandês-Zebu em lactação. Revista Brasileira de Zootecnia, 34(6), 2000-2008. https://doi.org/10.1590/S1516-35982005000600025

Baêta, F. C., \& Souza, F. C. (2010). Ambiência em edificações rurais: conforto animal. Universidade Federal de Viçosa.

Bandaranayaka, D. D., \& Holmes, C. W. (1976). Changes in the composition of milk and rumen contents in cows exposed to a high ambient temperature with controlled feeding. Tropical Animal Health and Production, 8(1), 38-46. https://doi.org/10.1007/BF02383364

Bertocchi, L., Vitali, A., Lacetera, N., Nardone, A., Varisco, G., \& Bernabucci, U. (2014). Seasonal variations in the composition of Holstein cow's milk and temperature-humidity index relationship. Animal: An International Journal of Animal Bioscience, 8(4), 667.

Borburema, J. B., Souza, B. B., Cezar, M. F., \& Pereira Filho, J. M. (2013). Influência de fatores ambientais sobre a produção e composição físico-química do leite. Agropecuária Científica No Semiárido, 9(4), 15-19. 
Borges, C. R. A., Azevedo, M., Lima, I. de A., Brasil, L. H. A., \& Ferreira, M. A. (2012). Heterogeneous genetic cows of three genetic groups in feedlot system in the state of Pernambuco, Brazil. Acta Scientiarum. Animal Sciences, 34(1), 91-96. https://doi.org/http://dx.doi.org/10.4025/actascianimsci.v34i1.11306.

Breihnholt, K. A., Gowen, F. A., \& Nwosu, C. C. (1981). Influence of environmental and animal factors on day and night grazing activity of imported Holstein-Friesian cows in the humid Lowland of Nigeria. Trop. Anim. Prod, 64, 300-307.

Collier, R. J., Dahl, G. E. \& Vanbaale, M. J. (2006). Major advances associated winth environmental effects on dairy cattle. Journal of Dairy Science. Champaing, 89, 1244-1253, doi: 10.3168/jds.S0022-0302(06)72193-2

Costa, M., Sant'Anna, A. C., \& Silva, L. C. M. (2015). Temperamento de bovinos Gir e Girolando: efeitos genéticos e de manejo. Inf. Agropecuário (Belo Horizonte), 36, 100-107.

Curtis, S. E. (1983). Environmental management in animal agriculture (Vol. 1). Iowa State University Press.

Damasceno, J. C., Baccari Júnior, F., \& Targa, L. A. (1998). Respostas fisiológicas e produtivas de vacas holandesas com acesso à sombra constante ou limitada. Revista Brasileira de Zootecnia, 27(3), 595-602.

Dash, S., Chakravarty, A. K., Singh, A., Upadhyay, A., Singh, M., \& Yousuf, S. (2016). Effect of heat stress on reproductive performances of dairy cattle and buffaloes: A review. Veterinary World, 9(3), 235-244. https://doi.org/10.14202/vetworld.2016.235-244

Davis M. S., Mader, T. L., Holt, S. M. \& Parhustt, A.M. (2003). Straregies to reduce feedlot cattle heat stress; Effect on tympanic temperature. Journal Animal Science, 81, 649-661. https://doi.org/10.2527/2003.813649x

Dhiman, T. R., \& Zaman, M. S. (2001). Desafios dos sistemas de produção de leite em confinamento em condições de clima quente. Simpósio de Nutrição e Produção de Gado de Leite, 2, 5-20.

Dobson, H., \& Smith, R. . (2000). What is stress, and how does it affect reproduction? Animal Reproduction Science, 60-61, 743-752. https://doi.org/10.1016/S0378-4320(00)00080-4

Ferreira, M. B. D., Lopes, B. C., \& Ferreira, J. J. (2001). Sustentabilidade do sistema de produção de leite com animais F1: perspectivas e pesquisa. MADALENA, FE,; MATOS LL de; HOLANDA JR.,. EV (Org.). Produção de Leite e Sociedade: Uma Análise Crítica Da Cadeia de Leite No Brasil. Belo Horizonte: FEPMVZ-Editora, 1, 383-404.

Ferreira, I. C., Martins, C. F., Fonseca Neto, A. M. \& Cumpa, H. C. B. (2017) Conforto térmico em bovinos leiteiros a pasto, 47 p- Planaltina, DF: Embrapa Cerrados. Disponível em < https://ainfo.cnptia.embrapa.br/digital/bitstream/item/178289/1/Doc-342.pdf>. 2011

Finocchiaro, R., van Kaam, J. B. C. H. M., Portolano, B., \& Misztal, I. (2005). Effect of Heat Stress on Production of Mediterranean Dairy Sheep. Journal of Dairy Science, 88(5), 1855-1864. https://doi.org/10.3168/jds.S0022-0302(05)72860-5

Fuquay, J. W. (1981). Heat Stress as it Affects Animal Production. Journal of Animal Science, 52(1), 164-174. https://doi.org/10.2527/jas1981.521164x

Hafez, E. S., Arnold, G. W., Bianca, W., Bogyo, T. P., Eskin, A., Folk, G. E., Gates, D. M., Hahn, L., Hensel, L., \& Hertig, B. A. (1973). Adaptación de los animales domésticos. Barcelona: Labor.

IBGE. (2017). Censo Agropecuário 2017.

Johnson, H. D. (1965). Environmental temperature and lactation (with special reference to cattle). International Journal of Biometeorology, 9(2), 103-116. https://doi.org/10.1007/BF02188466

Luz, L., Rodrigues, J. E. C., Ponte, F. C., \& Silva, C. N. (2013). Atlas geográfico escolar do estado do pará. Belém: GAPTA/UFPA.

Madalena, F. E., Peixoto, M. G. C. D., \& Gibson, J. (2012). Dairy cattle genetics and its applications in Brazil. Livestock Research for Rural Development, 24(6), 1-49.

Matarazzo, S. V., Perissinotto, M., Fernandes, S. A. A., Moura, D. J., \& Arcaro Jr, I. (2007). Eficiência de sistemas de climatização na área de descanso em instalações do tipo freestall e sua influência nas respostas produtivas e fisiológicas de vacas em lactação. Bolm Indústria Animal, 64, 221-232. 
Nääs, I. A., \& Arcaro Júnior, I. (2001). Influência de ventilação e aspersão em sistemas de sombreamento artificial para vacas em lactação em condições de calor. Revista Brasileira de Engenharia Agrícola e Ambiental, 5(1), 139-142. https://doi.org/10.1590/S141543662001000100026

Nascimento, S. T., Rossetto, Y. P., Silva, A. A., Mac-Lean, P. A. B., \& Tenório, J. P. L. (2017). Influência da temperatura ambiente no verão na produção de leite de vacas holandesas. Pubvet, 11(3), 217-223. https://doi.org/10.22256/pubvet.v11n3.217-223

Mollo Neto, Mario, Nääs, Irenilza de A., Carvalho, Victor C. de, \& Conceição, Antonio H. Q. (2014). Preventive diagnosis of dairy cow lameness. Engenharia Agrícola, 34(3), 577-589. https://dx.doi.org/10.1590/S0100-69162014000300020

Nogueira, S. S. (2010). Intensificação ou Diversificação? A pecuária leiteira em questão. Dissertação (Mestrado em Agriculturas Amazônicas) Núcleo de Ciências Agrárias e Desenvolvimento Rural, Universidade Federal do Pará. Pará.

Passetti, R. A. C., Eiras, C. E., Gomes, L. C., dos Santos, J. F., \& do Prado, I. N. (2016). Intensive dairy farming systems from holland and Brazil: SWOT analyse comparison. Acta Scientiarum - Animal Sciences, 38(4). https://doi.org/10.4025/actascianimsci.v38i4.31467.

Perissinotto, M., \& Moura, D. J. (2007). Evaluation of thermal comfort in dairy cattle using data mining. Revista Brasileira de Engenharia de Biossistemas, 1(2), 117. https://doi.org/10.18011/bioeng2007v1n2p117-126

Perissinotto, M., Moura, D. J., Cruz, V. F., Souza, S. R. L., Lima, K. A. O., \& Mendes, A. S. (2009). Conforto térmico de bovinos leiteiros confinados em clima subtropical e mediterrâneo pela análise de parâmetros fisiológicos utilizando a teoria dos conjuntos fuzzy. Ciência Rural, 39(5), 1492-1498. https://doi.org/10.1590/S0103-84782009005000094

Pires, M. D. F. A., \& Campos, A. T. (2004). Modificações ambientais para reduzir o estresse calórico em gado de leite. Embrapa Gado de Leite.

Rodrigues, L. R., Oliveira, Z. B., Azevedo, A., \& Dolianitis, B. M. (2017). Zoneamento Bioclimático para Vacas Leiteiras no Rio Grande Do Sul. VII Congresso Brasileiro de Biometeorologia, Ambiência, Comportamento e Bem-Estar Animal: Responsabilidade Ambiental e Inovação.

Santos, V. M. (2011). A economia do sudeste paraense = fronteira de expansão na periferia brasileira [Tese (doutorado) - Universidade Estadual de Campinas, Instituto de Economia, Campinas, SP]. http://www.repositorio.unicamp.br/handle/REPOSIP/286394

Sarmento, P. L. C. (2019). Zoneamento bioclimático para criação de ovinos no Estado do Pará. Trabalho de Conclusão de Curso (Graduação em Zootecnia) - Universidade Federal Rural da Amazônia, Belém.

Silva, R. G., Morais, D. A. E. F. \& Guilhermino, M. M. Evaluation of thermal stress indexes for dairy cows in tropical regions. Revista Brasileira de Zootencia, v. 36, n. 4, p. 1192-1198, 2007. http://dx.doi.org/10.1590/S1516-35982007000500028

Silva, I. J. O., Pandorfi, H., Acararo Jr., I., Piedade, S. M. S., \& Moura, D. J. (2002). Efeitos da climatização do curral de espera na produção de leite de vacas holandesas. Revista Brasileira de Zootecnia, 31(5), 2036-2042. https://doi.org/10.1590/S1516-35982002000800019

Silva, R. G. (2000). Introdução à bioclimatologia animal. Nobel.

Smith, D. L., Smith, T., Rude, B. J., \& Ward, S. H. (2013). Short communication: Comparison of the effects of heat stress on milk and component yields and somatic cell score in Holstein and Jersey cows. Journal of Dairy Science, 96(5), 3028-3033. https://doi.org/10.3168/jds.2012-5737

Soares, B. C., Lourenço Junior, J. B., Santos, M. A. S., Sena, A. L. S., Rodrigues Filho, J. A., Santana, A. C., Homma, A. K. O., Maciel e Silva, A. G., \& Andrade, S. J. T. (2019). Caracterização da cadeia produtiva da pecuária leiteira em Rondon do Pará, Pará, Brasil. Nucleus Animalium, 11(1), 25-37. https://doi.org/10.3738/21751463.3002

Soares, M. P., Gaya, L. G., Lorentz, L. H., Batistel, F., Rovadoscki, G. A., Ticiani, E., Zabot, V., Di Domenico, Q., Madureira, A. P., \& Pértile, S. F. N. (2011). Relationship between the magnitude of the inbreeding coefficient and milk traits in Holstein and Jersey dairy bull semen used in Brazil. Genetics and Molecular Research, 10(3), 1942-1947. 
Sota, R. L. (1996). Fisiología ambiental: mecanismos de respuesta del animal al estrés calórico. In: Jornada de Manejo Del Estress Calórico, 1., La Plata,.

Teke, B., \& Akdag, F. (2012). The effect of heat stress on some reproductive traits in Jersey cows under semi-humid conditions in Turkey. Bulg J Agric Sci, 18, 506-510.

West, J. W., Mullinix, B. G., \& Bernard, J. K. (2003). Effects of Hot, Humid Weather on Milk Temperature, Dry Matter Intake, and Milk Yield of Lactating Dairy Cows. Journal of Dairy Science, 86(1), 232-242. https://doi.org/10.3168/jds.S0022-0302(03)73602-9

Histórico do artigo:

Recebido: 28 de junho, 2020

Aprovado: 31 de julho, 2020.

Disponível online: 1 de novembro, 2020.
Licenciamento: Este artigo é publicado na modalidade Acesso Aberto sob a licença Creative Commons Atribuição 4.0 (CC-BY 4.0), a qual permite uso irrestrito, distribuição, reprodução em qualquer meio, desde que o autor e a fonte sejam devidamente creditados. 